\title{
Mycobacterial ESAT-6 and katG are Recognized by Sarcoidosis CD4+ T Cells When Presented by the American Sarcoidosis Susceptibility Allele, DRB1*1101
}

\author{
Kyra Oswald-Richter • Hiroe Sato • Rana Hajizadeh • \\ Bryan E. Shepherd • John Sidney • Alessandro Sette • \\ Lee S. Newman • Wonder Puryear Drake
}

Received: 12 August 2008 / Accepted: 29 May 2009/Published online: 18 June 2009

(C) The Author(s) 2009. This article is published with open access at Springerlink.com

\begin{abstract}
Introduction Genetic associations of American sarcoidosis susceptibility implicate MHC class II allele, DRB1*1101. We previously reported immune recognition of Mycobacterium peptides from peripheral cells of 26 sarcoidosis subjects, 24 PPD - healthy volunteers, and eight with latent tuberculosis infection.

Materials and Methods In order to further link these genetic and immunologic pillars of sarcoidosis pathogenesis, we performed flow cytometry on these same subjects to identify the cells responsible for immune responses to ESAT-6 and katG peptides, followed by HLA typing to determine allelic associations with recognition.
\end{abstract}

Kyra Oswald-Richter and Hiroe Sato contributed equally to this work.

\section{K. Oswald-Richter • R. Hajizadeh • W. P. Drake}

Department of Medicine, Division of Infectious Diseases,

Vanderbilt University School of Medicine,

Nashville, TN, USA

H. Sato

Clinical Genomics Group, Imperial College,

London, UK

\section{B. E. Shepherd}

Department of Biostatistics,

Vanderbilt University School of Medicine,

Nashville, TN, USA

\section{J. Sidney $\cdot$ A. Sette}

The La Jolla Institute for Allergy and Immunology,

La Jolla, CA, USA

\section{S. Newman}

Department of Preventive Medicine and Biometrics, University of Colorado at Denver \& Health Sciences Center,

Aurora, CO, USA
Discussion and Conclusion Sarcoidosis CD4+ T cells were primarily responsible for the systemic responses. Recognition was inhibited by monoclonal antibody against HLA-DR and HLA-DQ, but not HLA-DP. Immune recognition of ESAT-6 peptide NNALQNLARTISEAG was associated with possession of DRB $1 * 1101$. ESAT- 6 and katG presented by antigenpresenting cells expressing DRB $1 * 1101$-induced Th-1 responses from sarcoidosis $\mathrm{T}$ cells, thus providing a mechanistic insight for the association of HLA DRB1*1101 with sarcoidosis, and sarcoidosis $\mathrm{T}$ cell interaction with microbial antigens.

Keywords Sarcoidosis - MHC class II - Th-1 cytokines · CD4+ T cells

W. P. Drake

Department of Microbiology and Immunology,

Vanderbilt University School of Medicine,

Nashville, TN, USA

\author{
L. S. Newman \\ Department of Medicine, University of Colorado at \\ Denver \& Health Sciences Center, \\ Aurora, CO, USA
}

W. P. Drake $(\bowtie)$

Division of Infectious Diseases,

Vanderbilt University School of Medicine,

116121 st Avenue, AA2200 MCN,

Nashville, TN 37232, USA

e-mail: Wonder.drake@vanderbilt.edu 


\section{Introduction}

Studies of $\mathrm{T}$ cell receptor gene expression in sarcoidosis subjects reveal oligoclonal collections of $\alpha \beta^{+} \mathrm{CD} 4+\mathrm{T}$ cells, consistent with a MHC-restricted antigen-driven process [1-3]. This complex of antigen presented by MHC class II alleles is recognized by antigen-specific $\mathrm{T}$ cells, leading to the production of IL-2 and interferon gamma [IFN- $\gamma]$ [4]. Considerable evidence indicates that CD4+ T cells are important in the immunopathogenesis of sarcoidosis, and the development of granulomatous inflammation in the lung is associated with the accumulation of CD4 $+\mathrm{T}$ cells [5]. In the presence of antigen-presenting cells expressing MHC class II molecules, these T cells can be shown to proliferate and release Th-1 cytokines [5]. Certain HLA class II alleles have been associated with sarcoidosis outcome. HLA-DRB $1 * 03$ (previously DR3 or DR17) is strongly associated with symptoms found in acute sarcoidosis, and these features are linked to a favorable disease course [6-8]. Almost every DRB1*03-positive patient had a resolving disease within 2 years, and $50 \%$ of the DRB $1 * 03$-negative patients developed a non-resolving disease. In line with the association between DRB $1 * 03$ and the disease course was the finding of a significantly higher incidence of positive biopsies in the DRB1*03-negative patients, indicating a more widespread granuloma formation in these patients [8]. A more recent finding is that a particular CCR2 haplotype seems to associate, independently of DRB1*03, with Lofgren's syndrome [9]. In American sarcoidosis subjects, the HLA-DRB1*1101 allele was associated $(P \leq 0.01)$ with sarcoidosis in blacks and whites and had a population attributable risk of $16 \%$ in blacks and $9 \%$ in whites [10]. Among Polish sarcoidosis subjects, DRB $1 * 11$ has been associated with increased risk [11].

Recent studies have identified humoral and cellular immune responses to mycobacterial antigens among sarcoidosis subjects worldwide [12-14]. We recently reported cellular immune responses to mycobacterial virulence factors, ESAT-6 and katG among 15/26 sarcoidosis, compared to 1/24 PPD-negative (PPD-) and 7/8 PPDpositive ( $\mathrm{PPD}+$ ) subjects, due to latent tuberculosis using the enzyme-linked immunospot (ELISPOT) assays [13]. How the sarcoid antigen-specific CD4+ T cells interact with the $\mathrm{MHC} /$ mycobacteria peptide complex and the role of disease susceptible alleles in antigen presentation is currently unknown.

Epidemiologic evidence from sarcoidosis studies in multiple ethnic and racial groups suggests that HLA-DR and -DQ types confer risk or protection $[10,15,16]$. HLADRB $1 * 1101$ has been reported as an independent risk factor for disease among African American and Caucasian sarcoidosis subjects [10]. Since the ability to develop a
CD4+ $\mathrm{T}$ cell response in sarcoidosis depends on immunogenic peptides binding to HLA class II molecules, the primary aim of this study is to provide mechanistic insight into the genetic association of HLA DRB $1 * 1101$ with sarcoidosis susceptibility and the immunologic observation of Th-1 sarcoidosis immune responses to mycobacterial antigens. Using flow cytometry, we determined that CD4+ $\mathrm{T}$ cells were primarily responsible for the systemic sarcoidosis immune responses. In addition, we performed MHC class II typing on all study participants and discovered an association with immune responses to mycobacterial virulence factors and possession of the allele, HLA DRB $1 * 1101$.

\section{Methods}

Study Population This study was approved by the Vanderbilt University Institutional Review Board for human studies. Informed written consent was obtained from the study participant or their surrogates. All sarcoidosis subjects from the available patient database of the Vanderbilt University Pulmonary Clinic were invited to participate in the study. All patients came from the southeastern United States. For inclusion in this study, the clinical, histologic, and microbiologic criteria used to define sarcoidosis patients were as previously described [17]. Healthy PPDvolunteers must have undergone PPD testing by the Vanderbilt employee health services. PPD-positive subjects had written documentation of their PPD status, and had no evidence of active disease at the time of study enrollment. Sarcoidosis subjects underwent tuberculin skin testing at the discretion of their physician. Each sarcoidosis subject underwent a chart review to investigate the site of involvement by sarcoidosis. The demographic information of the study participants is listed in Table I.

Peptide Synthesis and Preparation of Peripheral Blood Mononuclear Cells (PBMC) The ESAT-6 and katG peptides were synthesized as previously described [13]. The sequence for ESAT-6 peptide 14 is NNALQNLARTISEAG. The sequence for katG peptide 13 is WTNTPTKWDNSFLEI. The preparation of PBMC also was as previously described [13].

Enzyme-Linked Immunospot Assay ELISPOT assays were performed as previously described [18]. The number of specific gamma interferon-secreting $\mathrm{T}$ cells was calculated by subtracting the mean negative control value from the mean spot-forming-cell (SFC) count for duplicate wells inoculated with peptide. Negative controls always had $<50$ SFC per $10^{6}$ input cells. A positive response was defined as a concentration of at least $50 \mathrm{SFC} / 10^{6} \mathrm{PBMC}$ that is at least 
Table I Immune Recognition and HLA-DR and HLA-DQ Type of Study Participants

\begin{tabular}{|c|c|c|c|c|c|}
\hline & $\operatorname{Age}^{\mathrm{a}} / \mathrm{race}^{\mathrm{b}} / \mathrm{sex}^{\mathrm{c}}$ & HLA type class II DR & HLA type class II DQ & $\operatorname{katG}^{\mathrm{d}}$ & ESAT- $^{\mathrm{d}}$ \\
\hline Sarcoidosis 1 & 58AAF & DRB1 0701/1101 & DQB1 0301/0303 & 580 & 100 \\
\hline Sarcoidosis 2 & 53AAM & DRB1 08/12 & DQB1 0402/0501 & 480 & 320 \\
\hline Sarcoidosis 3 & $50 \mathrm{CM}$ & DRB1 1001/1201 & DQB1 0501/0301 & 300 & 550 \\
\hline Sarcoidosis 4 & $55 \mathrm{CF}$ & DRB1 1101/1501 DRB3 0202 DRB5 0101 & DQB1 0301/0602 & 300 & 280 \\
\hline Sarcoidosis 5 & $52 \mathrm{AAF}$ & DRB1 1302/1503 DRB3 0301 DRB5 0202 & DQB1 0602/0609 & 110 & 500 \\
\hline Sarcoidosis 6 & 50AAM & DRB1 1101/1501 DRB3 0202 DRB5 0101 & DQB1 0602 & 90 & 130 \\
\hline Sarcoidosis 7 & 58AAM & DRB1 0701/1401 & DQB1 0202/0503 & 510 & $<50$ \\
\hline Sarcoidosis 8 & $52 \mathrm{AAF}$ & DRB1 0103/0701 & DQB1 0303/0501 & 500 & $<50$ \\
\hline Sarcoidosis 9 & $40 \mathrm{CM}$ & DRB1 0701/1301 DRB3 0202 & DQB1 0303/0603 & $<50$ & 590 \\
\hline Sarcoidosis 10 & $33 \mathrm{CF}$ & DRB1 0101/1501 & DQB1 0501/0602 & 420 & $<50$ \\
\hline Sarcoidosis 11 & $51 \mathrm{CF}$ & DRB1 0701/1101 & DQB1 0301/0303 & 370 & $<50$ \\
\hline Sarcoidosis 12 & 31AAM & DRB1 0302/0804 DRB3 0101 & DQB1 0301/0402 & 240 & $<50$ \\
\hline Sarcoidosis 13 & $33 \mathrm{CF}$ & DRB1 0301/1101 & DQB1 0301/09 & $<50$ & 100 \\
\hline Sarcoidosis 14 & 46AAM & DRB1 0701/1201 & DQB1 0202/0501 & 120 & $<50$ \\
\hline Sarcoidosis 15 & $62 \mathrm{CM}$ & DRB1 0404/1501 DBR5 0101 & DQB1 0302/0602 & 110 & $<50$ \\
\hline Sarcoidosis 16 & $47 \mathrm{CF}$ & DRB1 0401/1101 & DQB1 0301 & $<50$ & $<50$ \\
\hline Sarcoidosis 17 & 51AAF & DRB1 1101/1303 DRB3 0202 & DQB1 0201/0602 & $<50$ & $<50$ \\
\hline Sarcoidosis 18 & 42AAF & DRB1 7/15 & & $<50$ & $<50$ \\
\hline Sarcoidosis 19 & 49AAF & DRB1 1201/1503 DRB3 0101 DRB5 0101 & DQB1 0501/0602 & $<50$ & $<50$ \\
\hline Sarcoidosis 20 & $22 \mathrm{CM}$ & DRB1 1301 DRB3 0202 & DQB1 0603 & $<50$ & $<50$ \\
\hline Sarcoidosis 21 & $41 \mathrm{CF}$ & DRB1 0101/1302 & DQB1 0501/0609 & $<50$ & $<50$ \\
\hline Sarcoidosis 22 & 47AAM & DRB1 0804/1301 DRB3 0101 & DQB1 0501/0603 & $<50$ & $<50$ \\
\hline Sarcoidosis 23 & $47 \mathrm{CF}$ & DRB1 0101/1302 DRB3 0301 & DQB1 0501/0604 & $<50$ & $<50$ \\
\hline Sarcoidosis 24 & $51 \mathrm{CF}$ & DRB1 0401/0701 & DQB1 0201/0301 & $<50$ & $<50$ \\
\hline Sarcoidosis 25 & $37 \mathrm{CM}$ & DRB1 1301/1501 DRB3 0101 DRB5 0101 & DQB1 0602/0603 & $<50$ & $<50$ \\
\hline Sarcoidosis 26 & $61 \mathrm{CF}$ & DRB1 1302/1501 DRB3 0301 DRB5 0101 & DQB1 0602/0604 & $<50$ & $<50$ \\
\hline Control 1 & 36AAF & DRB1 0701/1101 & DQB1 0303/0301 & $<50$ & 380 \\
\hline Control 2 & $37 \mathrm{CM}$ & DRB1 1101/1501 & DQB1 0301/ 0602 & $<50$ & $<50$ \\
\hline Control 3 & $23 \mathrm{CM}$ & DRB1 0401/1101 DRB3 0202 & DQB1 0301 & $<50$ & $<50$ \\
\hline Control 4 & $58 \mathrm{CF}$ & DRB1 0301/1201 DRB3 0202 & DQB1 0201/0301 & $<50$ & $<50$ \\
\hline Control 5 & $25 \mathrm{CF}$ & DRB1 1201/1302 DRB3 0202/0301 & DQB1 0301/0609 & $<50$ & $<50$ \\
\hline Control 6 & $30 \mathrm{CM}$ & DRB1 0301 & DQB1 0201 & $<50$ & $<50$ \\
\hline Control 7 & 27AAM & DRB1 0701/1401 & DQB1 0201/ 0503 & $<50$ & $<50$ \\
\hline Control 8 & $33 \mathrm{CF}$ & DRB1 15/08 & DBQ1 0301/0609 & $<50$ & $<50$ \\
\hline Control 9 & $31 \mathrm{CM}$ & DRB1 0103/0701 & DQB1 0201/0301 & $<50$ & $<50$ \\
\hline Control 10 & $31 \mathrm{CF}$ & DRB1 15/08 & DQB1 0301/0602 & $<50$ & $<50$ \\
\hline Control 11 & $25 \mathrm{HM}$ & DRB1 0701/1501 DRB5 0101 & DQB1 0202/0602 & $<50$ & $<50$ \\
\hline Control 12 & $32 \mathrm{CM}$ & DRB1 0401/1501 DRB5 0101 & DQB1 0302/0602 & $<50$ & $<50$ \\
\hline Control 13 & $24 \mathrm{CF}$ & DRB1 0401/1501 DRB5 0101 & DQB1 0301/0602 & $<50$ & $<50$ \\
\hline Control 14 & $37 \mathrm{CF}$ & DRB1 0301 DRB3 0101 & DQB1 0201 & $<50$ & $<50$ \\
\hline Control 15 & $27 \mathrm{AAF}$ & DRB1 0701/1401 DRB3 0202 & DQB1 0201/0503 & $<50$ & $<50$ \\
\hline Control 16 & $33 \mathrm{AAF}$ & DRB1 0301/0701 DRB3 0202 & DQB1 0201 & $<50$ & $<50$ \\
\hline Control 17 & $33 \mathrm{CF}$ & DRB1 1301/1501 & DQB1 0602/ 0611 & $<50$ & $<50$ \\
\hline Control 18 & 31AAM & DRB1 0301/0302 DRB3 0101/0202 & DQB1 0201/0402 & $<50$ & $<50$ \\
\hline Control 19 & $51 \mathrm{CF}$ & DRB1 0404/1501 DRB5 0101 & DQB1 0302/0602 & $<50$ & $<50$ \\
\hline Control 20 & $35 \mathrm{CF}$ & DRB1 0301/1302 DRB3 0101/0301 & DQB1 0201/0604 & $<50$ & $<50$ \\
\hline Control 21 & $34 \mathrm{AAF}$ & DRB1 0701/1302 DRB3 0301 & DQB1 0501/0201 & $<50$ & $<50$ \\
\hline Control 22 & $34 \mathrm{CM}$ & DRB1 0701/1401 DRB3 0202 & DQB1 0201/0503 & $<50$ & $<50$ \\
\hline Control 23 & 37AAF & DRB1 0405/1503 DRB5 0101 & DQB1 0302/0602 & $<50$ & $<50$ \\
\hline
\end{tabular}


Table I (continued)

\begin{tabular}{|c|c|c|c|c|c|}
\hline & $\operatorname{Age}^{\mathrm{a}} / \operatorname{race}^{\mathrm{b}} / \operatorname{sex}^{\mathrm{c}}$ & HLA type class II DR & HLA type class II DQ & $\operatorname{katG}^{\mathrm{d}}$ & ESAT- $6^{\mathrm{d}}$ \\
\hline Control 24 & $32 \mathrm{AAF}$ & DRB1 0701/0901 & DQB1 0201/0303 & $<50$ & $<50$ \\
\hline $\mathrm{PPD}+1$ & $45 \mathrm{CF}$ & DRB1 0403/1101 & DQB1 0301/0302 & 400 & 500 \\
\hline $\mathrm{PPD}+2$ & 41AAF & DRB1 1101 DRB3 0202 & DQB1 0501/0602 & 750 & 230 \\
\hline $\mathrm{PPD}+3$ & $34 \mathrm{CF}$ & DRB1 0101/0301 & DQB1 0201/0501 & 430 & 400 \\
\hline $\mathrm{PPD}+4$ & 57AAM & DRB10701/1001 & DQB1 0202/0501 & 60 & 500 \\
\hline $\mathrm{PPD}+5$ & $49 \mathrm{CF}$ & DRB1 0404/1001 & DQB1 05010302 & 280 & $<50$ \\
\hline $\mathrm{PPD}+6$ & $63 \mathrm{CF}$ & DRB1 1501 & DQB1 0501/ 0602 & 180 & $<50$ \\
\hline $\mathrm{PPD}+7$ & $64 \mathrm{CF}$ & DRB1 1303/1401 & DQB1 0301/0503 & 80 & $<50$ \\
\hline $\mathrm{PPD}+8$ & $49 \mathrm{CF}$ & DRB1 0301 & DQB1 0201 & $<50$ & $<50$ \\
\hline
\end{tabular}

${ }^{\text {a }}$ Age in years

${ }^{\mathrm{b}} A A$ African American, $A$ African, $C$ Caucasian, $H$ Hispanic, $I$ Asian Indian

${ }^{\mathrm{c}} F$ female, $M$ male

${ }^{\mathrm{d}}$ Inteferon- $\gamma$ producing spot-forming units per million PBMC

three times higher than the background level. The research assistants were blind to the clinical diagnoses of the study participants throughout the analysis.

HLA II Typing Typing was performed at Dialysis Clinical Incorporated (Nashville, TN), which provides HLA typing for organ transplantion at Vanderbilt University Medical Center. The samples were typed for HLA-DRB1, DRB3, DRB5, and DQB1 using LabType (One Lambda) reverse Sequence-specific oligonucleotide probes (SSOP) and utilizing Luminex technology. Internal controls were included according to the guidelines of the American Society of Histocompatibility and Immunogenetics. Genomic DNA was first amplified using polymerase chain reaction (PCR) with a group-specific primer for each locus, biotinylated and detected using R-phycoerythrin-conjugated strepavidin (SAPE). Following denaturation and rehybridization to complementary DNA probes conjugated to fluorescently coded microspheres, the bead/PCR products were detected with SAPE. A flow analyzer, the LABScan 100, identified the fluorescent intensity of phycoerythrin (PE) on each microsphere. The assignment of HLA typing was based on the reaction pattern compared to with published HLA sequences. Analysis was performed using One Lambda software. Peptide binding assays to assess binding affinity of mycobacterial peptides with MHC class II alleles were performed at La Jolla Institute for Allergy and Immunology, La Jolla, CA by Dr. Alex Sette as previously described [19].

Cell Isolation and Culture The media used in all experiments was RPMI 1640 (cellgro) supplemented with 10\% fetal bovine serum (FBS; Gemini Bio-Products), penicillin (50 U/ml; cellgro), streptomycin (50 ug/ml; cellgro), sodium pyruvate ( $1 \mathrm{mM}$; cellgro), and glutamine $(2 \mathrm{mM}$; cellgro). To generate allogeneic dendritic cells (DCs), $\mathrm{CD}_{1} 4^{+}$monocytes were purified from Sarcoidosis 6 PBMC using the MACS ${ }^{\circledR}$ system (Miltenyi Biotec) and cultured in the presence of IL-4 $(100 \mathrm{ng} / \mathrm{ml})$ and GM-CSF $(50 \mathrm{ng} / \mathrm{ml}$; both from R\&D Systems) for 4-6 days. DCs were then matured by the addition of lipopolysaccharide (LPS) overnight. PBMC were isolated from sarcoidosis subject 6 and were activated with autogenic (self-derived) DCs and either ESAT-6 or katG peptides. The activated PBMC were then cultured in IL-2 containing medium for approximately 21 days.

Class II HLA Restriction Analysis by Intracellular Cytokine Staining of T Cells To identify IFN- $\gamma$ and IL-2-secreting T cells in response to ESAT-6, staining with a combination of $\mathrm{T}$ cell surface markers and intracellular staining was performed as previously described [20]. Briefly, 0.5-1.0× $10^{6}$ PBMC were incubated with $10 \mu \mathrm{M}$ ESAT- 6 and the antiCD28 and anti-CD49d mAbs $(1 \mu \mathrm{g} / \mathrm{ml}$ each; BD Biosciences) at $37^{\circ} \mathrm{C}$ under $5 \% \mathrm{CO}_{2}$ for $2 \mathrm{~h}$ before addition of $10 \mu \mathrm{g}$ of brefeldin A (Sigma) $/ \mathrm{ml}$. Following a 13-h incubation at $37^{\circ} \mathrm{C}$ under $5 \% \mathrm{CO}_{2}$, cells were washed and stained with the surface antibodies anti-CD8+ and anti-CD4+ (BD Biosciences) at $4^{\circ} \mathrm{C}$ for $30 \mathrm{~min}$. After washing, fixation, and permeabilization using Fix\&Perm Kit according to the manufacturer's instructions (Caltag, Burlingame, CA), antiIFN- $\gamma$ mAb (BD Biosciences) was added at $4^{\circ} \mathrm{C}$ for $30 \mathrm{~min}$. The lymphocyte population was identified using forward and $90^{\circ}$ light-scatter patterns, and fluorescence intensity was analyzed using a LSR II Multiparameter Cytometer (BD Biosciences Immunocytometry Systems). The presenting MHC class II molecules were determined by the addition of monoclonal antibodies ( $\mathrm{mAb}$ ) against HLA-DR, HLA-DP or HLA-DQ (BD Biosciences) at a concentration of $3 \mu \mathrm{g} / \mathrm{ml}$ $1 \mathrm{~h}$ prior to the addition of the ESAT- 6 to $0.5-1.0 \times 10^{6}$ 
PBMC. The experiment was then conducted as described above.

Immune Recognition with DRB1*1101, DRB3*0202 Transformed Cell Line Sweig cells are Epstein-Barr virusimmortalized lymphocyte cell lines (EBV-immortalized LCLs), derived by infecting adult B cells with the B958 strain. They were obtained as a generous gift of Janice Blum (Indiana University Purdue University Institute). The Sweig cells express HLA DRB1*1101 and DRB3*0202. Using the Sweig cells for antigen presentation, immune recognition of ESAT- 6 and $k a t G$ peptides were analyzed from the expanded cell lines for intracellular cytokine production of IFN- $\gamma$ by flow cytometry. Cells stimulated by staphylococcal enterotoxin B (SEB) served as the positive control, and unstimulated cells served as the negative control.

Statistical Analysis Comparisons of the distribution of $\mathrm{T}$ cell frequencies were performed using Kruskal-Wallis test (equivalent to the Wilcoxon rank sum test when comparing two groups). Categorical comparisons, such as immune reactivity to mycobacterial antigens by individuals within a group, were analyzed using Fisher's exact test. Multiple comparisons were performed. All performed comparisons are reported, all $p$ values are two-sided, and all analyses were performed using R (Version 2.1.1).

\section{Results}

Clinical and Demographic Characterization of Study Participants Sixty-one subjects had been previously tested for recognition of ESAT-6 and katG peptides: 26 sarcoidosis subjects, 24 PPD - healthy volunteers, eight subjects with latent tuberculosis infection, and three subjects who had undergone BCG vaccination. Of the sarcoidosis subjects, $46 \%$ were African Americans, $42 \%$ were male, and $58 \%$ less than or equal to 50 years of age. Of the PPDpatients, 33\% were African American, 4\% were Hispanic, $38 \%$ were male and $88 \%$ less than or equal to 50 years of age (Table I). Of the eight subjects with latent tuberculosis infection, 25\% were African American, 13\% male, and $63 \%$ less than or equal to 50 years of age (Table I). None of the sarcoidosis subjects who underwent tuberculin skin testing were positive.

Immune Recognition of katG and ESAT-6 Peptides Reveals a Significant Difference in the T Cell Frequencies Among $P P D^{-}$, Sarcoidosis, and PPD+ Subjects Thirteen of the 26 sarcoidosis subjects recognized katG peptide 13 compared to none of the PPD - control subjects $(p<0.0001$, two-tailed
Fisher's; Table I). Seven of the eight subjects with latent tuberculosis infection (PPD+ 1-8) displayed immune recognition to $\mathrm{katG}$ peptide. Eight of 26 sarcoidosis subjects, one of 24 control subjects ( $p=0.024$, two-tailed Fisher's), and four of eight PPD+ subjects ( $p=0.41$, twotailed Fisher's; Table I) recognized ESAT-6 peptide14.

FACS Analysis Reveals that CD4+ T Cells are Primarily Responsible for the Sarcoidosis Response to Mycobacterial Antigens Previous work has demonstrated the production of IL-2 and IFN- $\gamma$ by sarcoidosis PBMC in response to ESAT-6 and katG, using ELISPOT analysis [13]. Flow cytometry revealed that production of the Th-1 cytokines were primarily from $\mathrm{CD} 4+\mathrm{T}$ cells. While recognition by $\mathrm{CD} 4+$ and $\mathrm{CD} 8+\mathrm{T}$ cells was detected among some latent tuberculosis subjects (data not shown), CD4 $+\mathrm{T}$ cells responses were mainly detected among the sarcoidosis subjects to ESAT-6. Sarcoidosis 6 demonstrated CD4+ and CD8+ T cell responses to ESAT-6 (Fig. 1a). CD4+ T cell recognition of katG was also detected; a representative analysis of Sarcoidosis 10 is shown in Fig. $1 b$.

HLA-DR and HLA-DQ Inhibits Recognition of Mycobacterial Peptides After the observance of mainly CD4+ T cell responses to mycobacterial peptides, we investigated for HLA class II restriction of immune recognition of ESATpeptide. Using flow cytometry, we examined the effects of mAbs against HLA-DR, -DQ, and -DP on antigen presentation of ESAT-6 and katG (Fig. 2). Immune recognition of ESAT-6 peptide was partially inhibited after blocking antigen presentation with anti-HLA-DR or antiHLA-DQ mAb. Monoclonal antibody against HLA-DP had no effect.

\section{Immune Recognition of ESAT-6 Peptide 14 is Associated with DRB1*1101}

Possessing the HLA-DRB $1 * 1101$ allele has been associated with increased risk of susceptible disease in Caucasian and African American sarcoidosis subjects [10]. There was no significant difference in the frequency of the allele DRB1*1101 among the sarcoidosis, PPD- or PPD+ groups. We assessed for HLA associations with antigen recognition for ESAT-6 peptide 14 among the study participants. A total of 13 subjects from all three groups (sarcoidosis, PPD- and PPD+ due to latent MTB infection) recognized ESAT-6 peptide 14 (Table I). Seven of the 13 subjects recognizing ESAT-6 peptide 14 possessed the DRB1*1101 allele. In contrast, 45 subjects did not recognize ESAT-6 peptide 14. Only five of those 45 subjects possessed the DRB1*1101 allele. The presence of 
Fig. 1 CD4+ T cells are primarily responsible for the sarcoidosis systemic response to mycobacterial antigens. Flow cytometry revealed sarcoidosis CD4+ T cells were primarily responsible for peripheral IL-2 and interferon- $\gamma$ production when stimulated with ESAT-6 peptides. While CD8+ T cell responses were identified by flow cytometry among subjects with latent tuberculosis, these responses were rare among sarcoidosis subjects. In one sarcoidosis subject (Sarcoidosis 6), CD4+ and CD8+ T cell responses were significantly elevated above baseline (a). In Sarcoidosis 10 , stimulation with katG peptide also induced strong CD4+ T cells response (b)

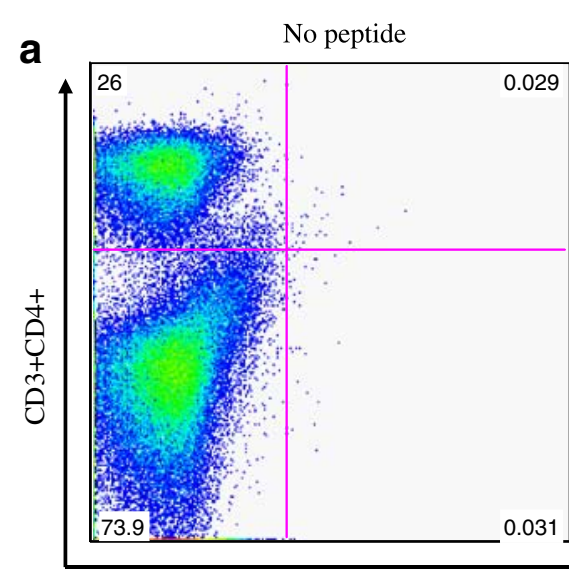

ESAT-6 peptide 14
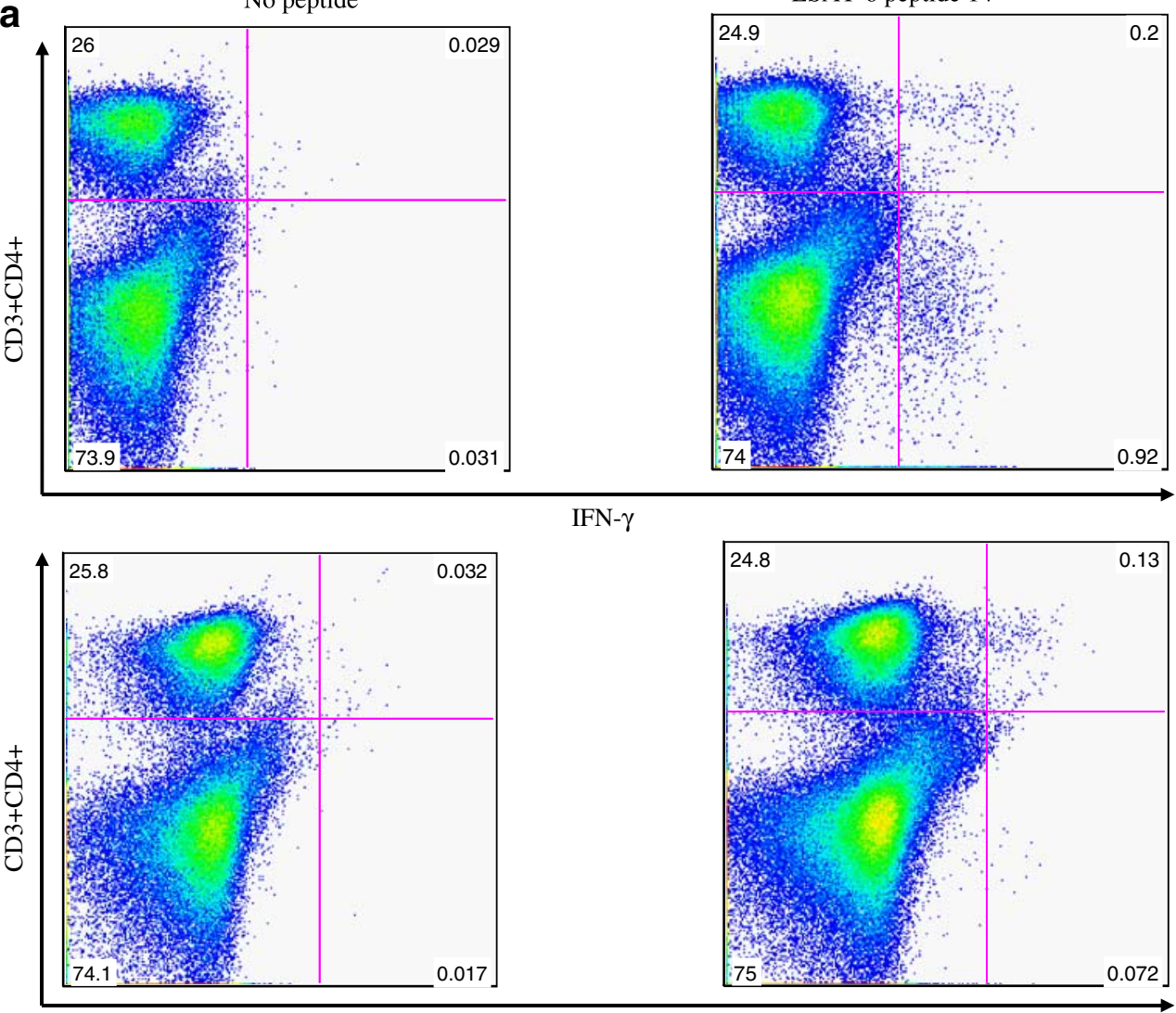

IL-2

b
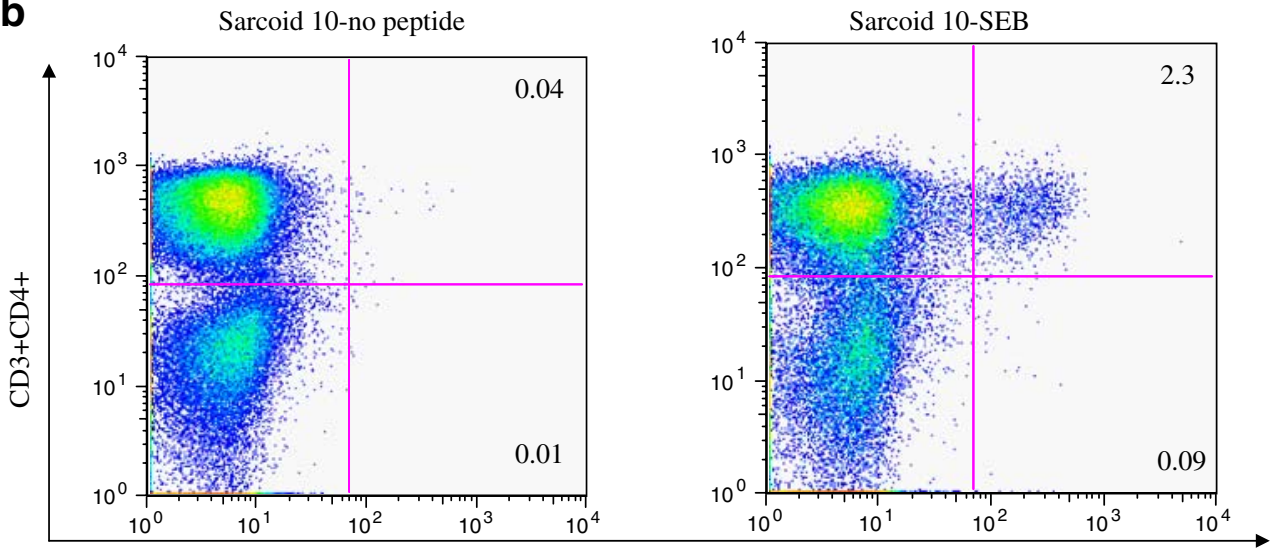

IL-2

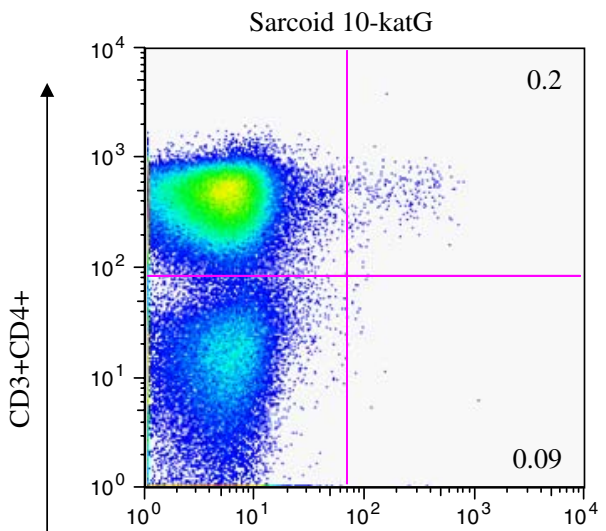




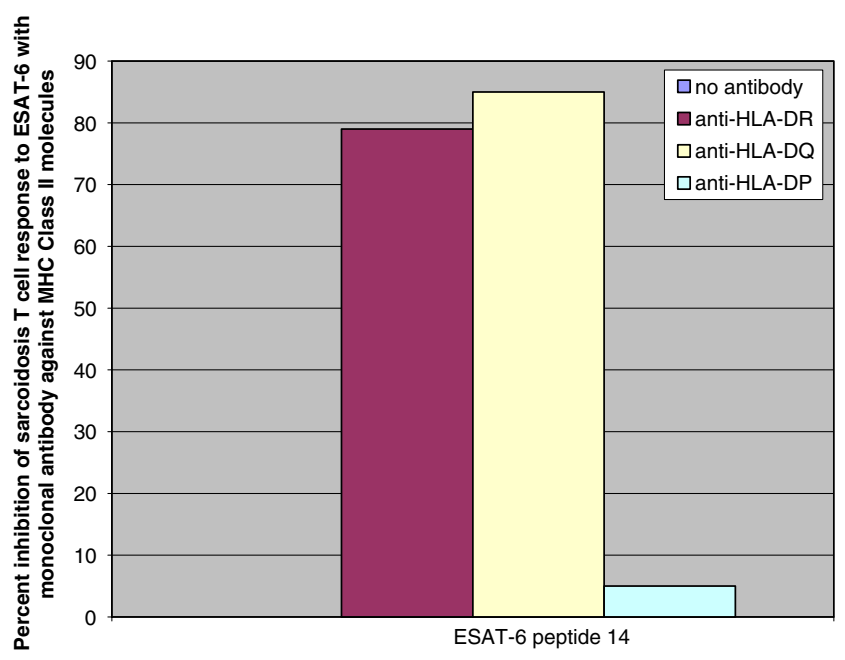

Fig. 2 CD4+ T cell responses are partially blocked by anti-HLA-DR and anti-HLA-DQ antibodies. Consistent with independent reports from other labs, immune responses to ESAT- 6 and katG were blocked with monoclonal antibody against HLA-DR. Partial inhibition of immune recognition also was observed with monoclonal antibody against HLA-DQ. HLA-DP did not appear to have a role in ESAT-6 antigen presentation

DRB1*1101 in seven of 13 subjects recognizing ESAT-6 peptide 14, compared to five of 45 who did not, is significantly distinct ( $p=0.003$, two-tailed Fisher's), and suggests that DRB1*1101 could be associated with antigen presentation of ESAT-6 peptide 14.

Because the frequency of HLA alleles varies according to ethnicity, we assessed allelic associations with immune recognition of these mycobacterial peptides by ELISPOT among the 22 African Americans and 35 Caucasians subjects in this study. The lone Hispanic subject (Control 11) was omitted from the analysis due to the lack of Hispanic subjects in the other groups. Of the African American subjects from all three groups (sarcoidosis, PPDand PPD + due to latent TB), a total of seven demonstrated reactivity to ESAT-6 peptide 14; four of the seven possessed the DRB $1 * 1101$ allele (Sarcoidosis 1, 6, Control 1 , PPD+ 2). This is in contrast to DRB $1 * 1101$ being present in only one (Sarcoidosis 17) of the 15 African Americans who did not recognize ESAT-6 peptide $14(p=$ 0.02, two-tailed Fisher's; Table I, Fig. 3). Of the six Caucasians who recognized ESAT-6 peptide 14, three possessed DRB1*1101 (Sarcoidosis 4, 13, PPD+ 1); in contrast, of the 29 Caucasians who did not recognize this peptide, four possessed DRB1*1101 (Sarcoidosis 11, 16, Control 2, 3; $p=0.08$, two-tailed Fisher's; Table I, Fig. 3). There were no allelic associations with immune recognition of katG peptides (Table I).

Sarcoidosis T Cells Recognize ESAT-6 and katG Antigens Presented by DRB1*1101-Expressing Antigen-Presenting Cells The observation that monoclonal antibody against HLA-DR partially inhibited immune responses to ESAT-6, along with the detection of immune responses to ESAT-6 and $\mathrm{katG}$ in a subject homozygous for DRB1*1101 (PPD+ 2) suggested that the peptides could be presented by this allele. Using the Sweig cell line which presents using the DRB $1 * 1101$ allele, we assessed for immune responses to
Fig. 3 Immune recognition of ESAT-6 peptide 14 by sarcoidosis subjects according to HLADRB1*1101. The DRB1*1101 allele has a positive association with risk of sarcoidosis. Of 13 subjects recognizing ESAT-6 peptide 14 , seven possessed the HLA-DRB $1 * 1101$ allele, compared to five of 45 non-responders ( $p=0.0003$ ). Controlling for ethnicity, this difference continued to be significant for African Americans, in which four of the seven who recognized this peptide possessed the HLADRB1*1101, compared to one of 15 who did not $(p=0.02)$. Among the Caucasian subjects, there was a trend (three out of six compared to four out of 29) toward significance $(p=0.08)$

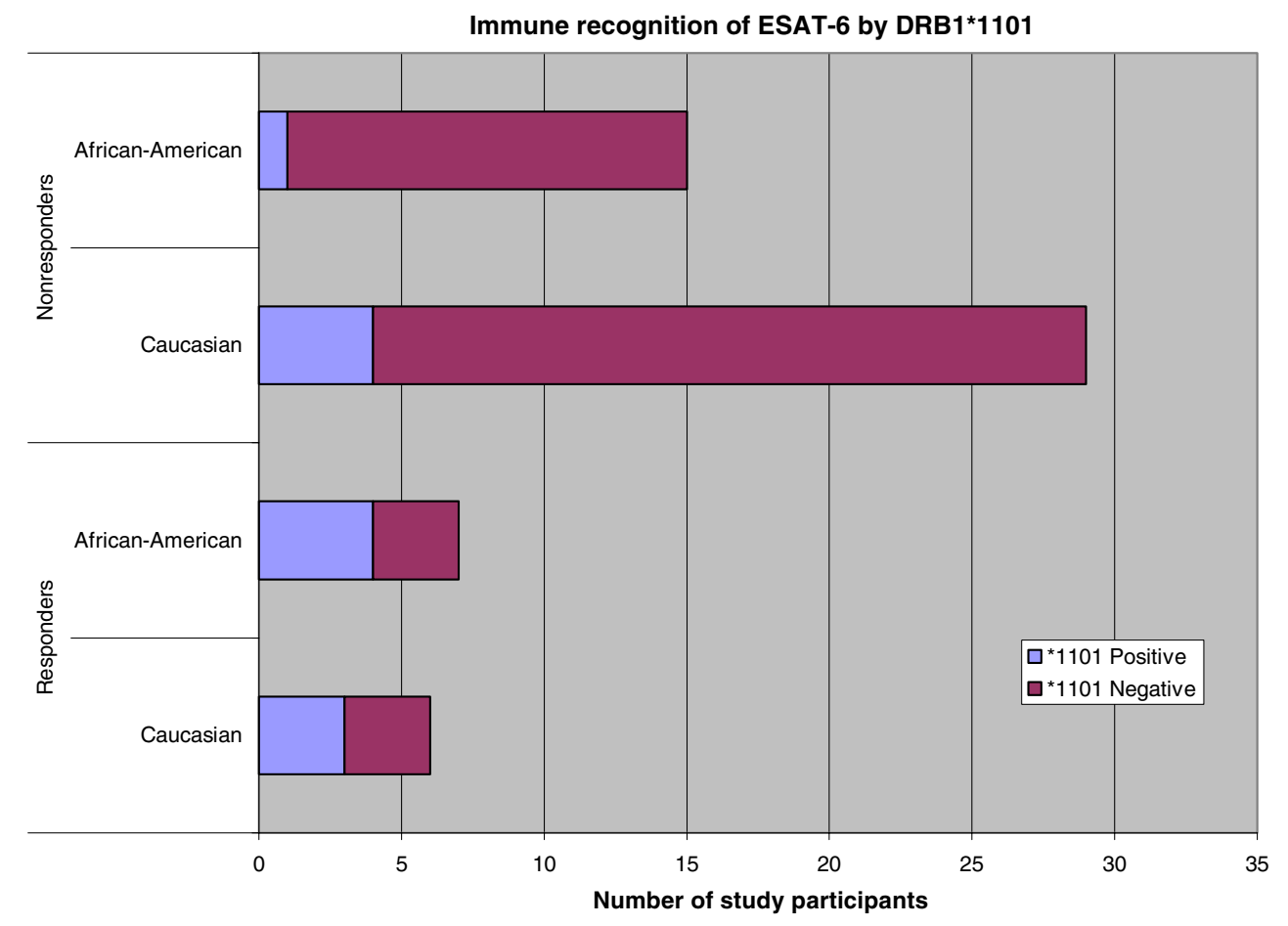


ESAT-6 and katG peptides from an expanded cell line of Sarcoidosis 6. This subject had the MHC class II alleles DRB1*1101/1501. The expanded cell line was generated from PBMC stimulated with ESAT-6 peptide 14 or katG peptide 13. The expanded ESAT-6 line demonstrated no response to ESAT-6 peptide 14 without the presence of Sweig cells, confirming the loss of the patient's own antigen-presenting cells during expansion. The addition of Sweig cells and ESAT-6 peptide to the ESAT-6 expanded cell line displayed a strong CD4+ IFN- $\gamma$ response (Fig. 4). The ESAT-6 expanded cell line stimulated with katG resulted in a minimal response. The katG-expanded cell line resulted in minimal response, after stimulation with ESAT-6. It was noteworthy also that PBMC expanded with katG peptide 13 also demonstrated strong CD4+ responses to katG peptides if presented by the Sweig cells. This data confirms that mycobacterial virulence factors ESAT- 6 and katG can be presented by the sarcoidosis susceptibility allele, DRB $1 * 1101$.

\section{Discussion}

This study demonstrates, for the first time, that the antigenic response in sarcoidosis is associated with HLA type. Notably, with an HLA-DR genotype that is known to confer increased risk for sarcoidosis in both US Caucasians and African Americans. These are important steps in the line of evidence supporting the hypothesis that mycobacterial antigens may have a role in sarcoidosis pathogenesis by inducing an adaptive granulomatous response in genetically susceptible individuals. It is also probable that other HLADR alleles are important in immune recognition. Studies of associations between HLA type and recognition of ESAT-6 among tuberculosis patients have noted that individual epitopes can be presented by diverse HLA types to human $\mathrm{T}$ cells, as well as that a given HLA type can present distinct epitopes [21, 22]. Our findings support earlier evidence of humoral responses to mycobacterial antigens $[12,23]$, and with the published literature regarding HLA specificity for mycobacterial antigens in tuberculosis and leprosy - two kindred granulomatous disorders [24-27].

The importance of the sarcoidosis CD4+ T cell response has been previously reported in sarcoidosis BAL [5]. Flow analysis confirmed that $\mathrm{CD} 4+\mathrm{T}$ cells were primarily responsible for the systemic sarcoidosis immune responses to katG and ESAT-6 peptides. In only one of the sarcoidosis subjects was a systemic CD8 $+\mathrm{T}$ cell response noted (Fig. 1). Consistent with the vigorous immune responses detected by ELISPOT analysis, flow cytometry revealed that in some subjects $\sim 1 \%$ of the systemic CD4+ T cells recognized mycobacterial antigens (Fig. 1). In the PPD+ subjects, CD4+ and CD8+ T cell responses were noted by

DRB1*1101 Antigen-presenting cell line-

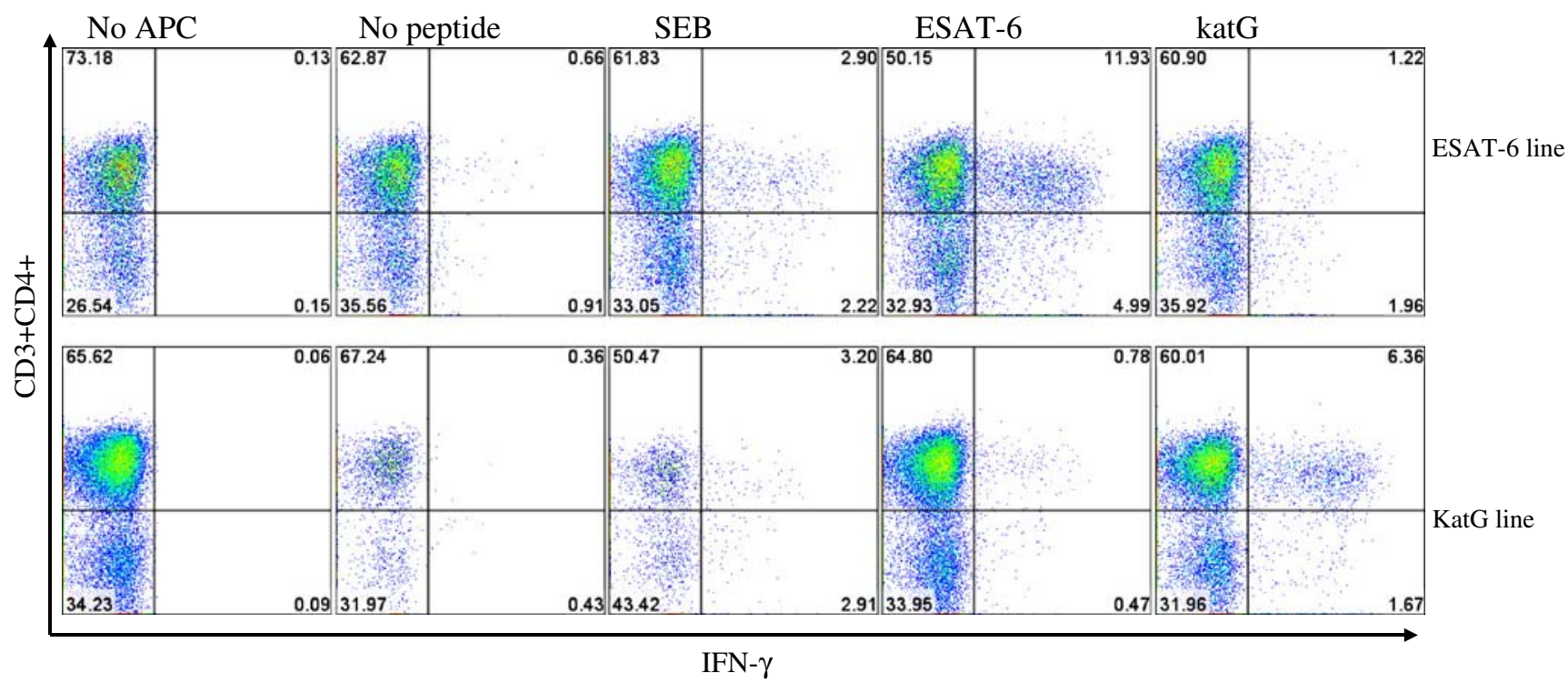

Fig. 4 HLA-DRB1*1101 presents ESAT-6 to sarcoidosis CD4+ T cells. Intracellular cytokine staining for IFN- $\gamma$ was performed on expanded ESAT- 6 and katG cell lines derived from sarcoidosis subject 6, after stimulation with either ESAT-6 peptide 14, katG peptide 13, or SEB. No recognition was observed in the expanded cell lines alone, demonstrating the absence of baseline IFN- $\gamma$ production. Expanded cells stimulated with ESAT-6 or katG peptide in the absence of Sweig cells revealed minimal responses, confirming the loss of antigenpresenting cells during expansion. Significant responses to ESAT-6 and $\mathrm{katG}$ were observed in the respective cell line when antigen was presented using the DRB1*1101 expressing Sweig cell lines. Shown are representative flow cytometry dot plots indicating percentage of sarcoidosis $\mathrm{CD} 4+\mathrm{T}$ cell responses to the different stimulation conditions 
flow cytometry (data not shown). The inhibition ESAT-6 recognition by HLA-DR and HLA-DQ but not HLA-DP is consistent with prior reports of antigen presentation of ESAT-6 epitopes [21, 22]. Studies of HLA associations with tuberculosis and lepromatous antigens reveal that most antigens are recognized in association with HLA-DR molecules (serologically defined as HLA-DR1 to HLADR10) [24-27]. HLA molecules encoded by HLA-DRB3 (HLA-DR52) and HLA-DRB4 (HLA-DR53) are also noted to be important in presentation of antigens from Mycobacterium tuberculosis and Mycobacterium leprae to Th-1 cells [27].

Our observation of an association between ESAT-6 response and HLA-DRB1*1101 is noteworthy because (1) it is consistent with the epidemiologic data showing increased sarcoidosis risk, and (2) it is consistent with experimental evidence in tuberculosis and leprosy. The observation of HLA class II associations between ESAT-6 and HLA DRB1*1101has been previously reported in tuberculosis subjects [24]. In addition, Mustafa et al. noted two other serologic HLA associations with this peptide sequence: HLA-DR2 (DRB1*15, 16) and HLA-DQ2 (DQB1*0201 and DQB1*0202). Because the Sweig cell line also presents $\mathrm{DRB} 3 * 0202$, it is possible that the peptides are presented by this allele, or even by HLA-DQ, although this is less likely given the lack of recognition without the Sweig cell line. On the converse side, there have been numerous reports of HLA associations with sarcoidosis, one of the largest of which was conducted in the United States, the ACCESS (A Case-Controlled Etiologic Study of Sarcoidosis) study [19]. This study noted a positive association between HLA-DRB1*1101 and African American and Caucasian sarcoidosis subjects. The observation of an association of HLA DRB1*1101 with immune recognition of ESAT-6 peptide 14 provides a possible immunologic and genetic basis of why this allele, as well as others, may be important in some sarcoidosis subjects. When assessing for the binding capacity of ESAT6 peptide 14 to DRB1*1101, it is consistent with moderate to weak binding capacity [DRB1*1101 (2,358 nm) and DRB1*1201 (4,416 nm)]. Epitopes have been identified in peptides with binding capacities ranging between 1,000$5,000 \mathrm{~nm}$ (John Sidney, personal communication). ESAT-6 peptide 14 demonstrated the strongest binding to DRB1*0101 (2.7 nm). No significant binding of KatG peptide 13 to HLA-DRB1*1101 was observed $(25,613 \mathrm{~nm})$; binding affinities greater than 5,000 are not considered significant (John Sidney, personal communication).

These findings do not suggest that this mycobacterial antigen and DRB1*1101 solely are the most important factor for the development of sarcoidosis. PBMC from the lone PPD- subject (Control 1) that did recognize ESAT-6 peptide 14 possessed DRB1*1101, but had no evidence of sarcoidosis. There were PBMC of sarcoidosis subjects that did not possess the DRB $1 * 1101$ allele yet recognized mycobacterial antigens (Sarcoidosis 2, 5, 9, for example; Table I). Also, there were PBMC from sarcoidosis persons which did not recognize either mycobacterial antigen (Sarcoidosis 16-26).

Although we did not find specific HLA associations with immune recognition of katG peptides, use of the DRB1*1101 Sweig cell line suggests that this epitope could be presented by DRB1*1101. This imperfect association between the presence of the HLA class II alleles and peptide recognition is likely due to the known degenerate binding ability of HLA class-II restricted peptides [28].

This detection of antigen-specific immune recognition to ESAT-6 and katG peptides contributes to the previously reported evidence of mycobacterial antigens as a cause of sarcoidosis. As previously reported, sarcoidosis may have more than one etiology [29], which is influenced by the host's HLA type, i.e., one's genetic predisposition to present and recognize particular antigens than can trigger granuloma formation. These findings suggest that in a subset of genetically susceptible sarcoidosis patients' immune responses to mycobacterial antigens may serve as the initial stimulus for the development of disease. In future studies that purport to show whether microbial antigen(s) are associated with sarcoidosis, investigation of genetic risk factors contributing to risk will be important, in order to explain why some patients are found to have immune responses to microbial antigens and others are not. Future studies will involve longitudinal assessment of immune reactivity to these antigens in newly diagnosed sarcoidosis subjects, as well as studies of immune reactivity to these antigens in matching cases and controls with respect to the presence of HLA alleles.

\section{Conclusions}

The observation of familial clustering and racial differences in sarcoidosis disease prevalence supports the hypothesis that genetics have an important role in sarcoidosis pathogenesis. A multicenter trial conducted in the United States, ACCESS, identified an association of the MHC class II allele, DRB1*1101 with increased susceptibility to sarcoidosis. CD4+ T cell responses to antigen presented in context of MHC class II alleles is thought to be the core of sarcoidosis pathogenesis. Here, we demonstrate that CD4+ $\mathrm{T}$ cells are primarily responsible for the observed systemic responses and that recognition is associated with possession of DRB $1 * 1101$. We also demonstrate that the mycobacterial virulence factors, ESAT- 6 and $\mathrm{katG}$, when presented by DRB1*1101 are recognized by sarcoidosis CD4+ T cells. These findings suggest that in a subset of genetically 
susceptible sarcoidosis patients' immune responses to mycobacterial antigens may serve as the initial stimulus for the development of disease. Our study provides new insights into the mechanisms underlying MHC class II genetic associations with sarcoidosis, and the interaction of sarcoidosis CD4+ T cells with bacterial virulence factors.

Acknowledgments This work was supported by RWJF 041300; HLBI R21077460; R01 HL 83839; R01 AI 65744; 5 M01 RR 00095. We would like to thank Dr. Wilfred Ajayi and Vanderbilt Pediatric Immunology Core for technical assistance with this project.

Conflict of Interests The authors have declared that no conflicts of interests exist.

Open Access This article is distributed under the terms of the Creative Commons Attribution Noncommercial License which permits any noncommercial use, distribution, and reproduction in any medium, provided the original author(s) and source are credited.

\section{References}

1. Robinson BW, McLemore TL, Crystal RG. Gamma interferon is spontaneously released by alveolar macrophages and lung Tlymphocytes in subjects with pulmonary Sarcoidosis. J Clin Invest. 1985; 75:1488-95.

2. Moller DR, Forman JD, Liu MC, Noble PW, Greenlee BM, Vyas $\mathrm{P}$, et al. Enhanced expression of IL-12 associated with Th1 cytokine profiles in active pulmonary sarcoidosis. J Immunol. 1996;156:4952-60.

3. Greene CM, Meachery G, Taggart CC, Rooney CP, Coakley R, O'Neill SJ, et al. Role of IL-18 in CD4+ T lymphocyte activation in Sarcoidosis. J Immunol. 2000;165:4718-24.

4. Saltini C, Spurzem JR, Lee JJ, Pinkston P, Crystal RG. Spontaneous release of IL-2 by lung $\mathrm{T}$ lymphocytes in active pulmonary sarcoidosis in primarily from the Leu $3+\mathrm{DR}+\mathrm{T}$ cell subset. J Clin Invest. 1986;77:1962-70.

5. Grunewald J, Eklund A. Role of CD4+ T cells in sarcoidosis. Proc Am Thorac Soc. 2007;4(5):461-4.

6. Hedfors E, Lindstrom F. HLA-B8/DR3 in sarcoidosis: correlation to acute onset disease with arthritis. Tissue Antigens. 1983;22: 200-203.

7. Gardner J, Kennedy HG, Hamblin A, Jones E. HLA associations in sarcoidosis: a study of two ethnic groups. Thorax. 1984;39:19-22.

8. Grunewald J, Eklund A. Löfgren's syndrome: human leukocyte antigen strongly influences the disease course. Am J Respir Crit Care Med. 2009;179(4):307-12.

9. Spagnolo P, Sato H, Grunewald J, Brynedal B, Hillert J, Mana J, et al. A common haplotype of the $\mathrm{C}-\mathrm{C}$ chemokine receptor 2 gene and HLA-DRB $1 * 0301$ are independent genetic risk factors for Lofgren's syndrome. J Intern Med. 2008;264:433-41.

10. Rossman MD, Thompson B, Frederick M, Maliarik M, Iannuzzi MC, Rybicki BA, et al. HLA-DRB $1 * 1101$ : a significant risk factor for sarcoidosis in blacks and whites. Am J Hum Genet. 2003;73:720-35.

11. Dubaniewicz A, Dubaniewicz-Wybieralska M, Moszkowska G, Sternau A, Dubaniewicz A. Comparative analysis of DR and DQ alleles occurrence in sarcoidosis and tuberculosis in the same ethnic group: preliminary study. Sarcoidosis Vasc Diffuse Lung Dis. 2006;23(3):180-9.

12. Song Z, Marzilli L, Greenlee BM, Chen ES, Silver RF, Askin FB, et al. Mycobacterial catalase-peroxidase is a tissue antigen and target of the adaptive immune response in systemic Sarcoidosis. J Exp Med. 2005;201:755-67.

13. Drake WP, Dhason MS, Nadaf M, Shepherd BE, Vadivelu S, Hajizadeh R, et al. Cellular recognition of Mycobacterium tuberculosis ESAT-6 and KatG peptides in systemic sarcoidosis. Infect Immun. 2007;75(1):527-30.

14. Dubaniewicz A, Trzonkowski P, Dubaniewicz-Wybieralska M, Dubaniewicz A, Singh M, Myśliwski A. Mycobacterial heat shock protein-induced blood T lymphocytes subsets and cytokine pattern: comparison of sarcoidosis with tuberculosis and healthy controls. Respirology. 2007;12(3):346-54.

15. Satoe H, Grutters JC, Pantelidis P, Mizzon N, Ahmad T, van Houte A-J, et al. HLA-DQB $1 * 0201$ : a marker for good prognosis in British and Dutch patients with Sarcoidosis. Am J Respir Cell Mol Biol. 2002;27:406-12.

16. Iannuzzi MC, Maliaric MJ, Poisson LM, Rybicki BA. Sarcoidosis susceptibility and resistance in HLA-DQB1 alleles in African Americans. Am J Respir Crit Care Med. 2003;167:1225-31.

17. Drake WP, Pei Z, Pride DT, Collins RD, Cover TL, Blaser MJ. Molecular analysis of sarcoidosis tissues for mycobacterium species DNA. Emerg Infect Dis. 2002;8(11):1334-41.

18. Kaufmann DE, Bailey PM, Sidney J, Wagner B, Norris PJ, Johnston $\mathrm{MN}$, et al. Comprehensive analysis of human immunodeficiency virus type 1-specific CD4 responses reveals marked immunodominance of gag and nef and the presence of broadly recognized peptides. J Virol. 2004;78(9):4463-77.

19. Sidney J, Southwood S, Oseroff C, del Guercio MF, Sette A, Grey HM. Measurement of MHC/peptide interactions by gel filtration. Curr Protoc Immunol. 2001;18:3.

20. Goulder PJ, Tang Y, Brander C, Betts MR, Altfeld M, Annamalai $\mathrm{K}$, et al. Functionally inert HIV-specific cytotoxic T lymphocytes do not play a major role in chronically infected adults and children. J Exp Med. 2000;192:1819-32.

21. Shams H, Klucar P, Weis SE, Lalvani A, Moonan PK, Safi $\mathrm{H}$, et al. Characterization of a Mycobacterium tuberculosis peptide that is recognized by human CD4+ and CD8+ T cells in the context of multiple HLA alleles. J Immunol. 2004;173(3): 1966-77.

22. Mustafa AS, Shaban FA, Al-Attiyah R, Abal AT, El-Shamy AM, Andersen P, et al. Human Th1 cell lines recognize the Mycobacterium tuberculosis ESAT-6 antigen and its peptides in association with frequently expressed HLA class II molecules. Scand J Immunol. 2003;57(2):125-34.

23. Dubaniewicz A, Kampfer S, Singh M. Serum anti-mycobacterial heat shock proteins antibodies in Sarcoidosis and tuberculosis. Tuberculosis. 2006;6(1):60-7.

24. Lombard Z, Dalton DL, Venter PA, Williams RC, Bornman L. Association of HLA-DR, -DQ, and vitamin D receptor alleles and haplotypes with tuberculosis in the Venda of South Africa. Hum Immunol. 2006;67(8):643-54.

25. Oftung F, Shinnick TM, Mustafa AS, Lundin KEA, Godal T, et al. Heterogeneity among T-cell clones recognizing an HLA-DR4, Dw4-restricted epitope from the $18 \mathrm{kDa}$ antigen of Mycobacterium leprae defined synthetic peptides. J Immunol. 1990;144:1478.

26. Geluk A, Ottenhoff TH. HLA and leprosy in the pre and postgenomic eras. Hum Immunol. 2006;67(6):439-45.

27. Borrás SG, Cotorruelo C, Racca L, Recarte M, Garcías C, Biondi $\mathrm{C}$, et al. Association of leprosy with HLA-DRB1 in an Argentinean population. Ann Clin Biochem. 2008;45(Pt 1):96-8.

28. Valli A, Sette A, Kappos L, Oseroff C, Sidney J, Miescher G, et al. Binding of myelin basic protein peptides to human histocompatibility leukocyte antigen class II molecules and their recognition by $\mathrm{T}$ cells from multiple sclerosis patients. J Clin Invest. 1993;91(2):616-28.

29. Iannuzzi MC, Rybicki BA, Teirstein AS. Sarcoidosis. N Engl J Med. 2007;357:2153. 